EPJ Web of Conferences 59, 09003 (2013)

DOI: $10.1051 /$ epjconf/20135909003

(C) Owned by the authors, published by EDP Sciences, 2013

\title{
Nonlinear effects of beam-plasma instabilities on neutralized propagation of intense ion beams in background plasma
}

\author{
Edward A. Startseva , Igor Kaganovich and Ronald C. Davidson \\ Princeton Plasma Physics Laboratory, Princeton, New Jeresey 08543, USA
}

\begin{abstract}
The streaming of an intense ion beam relative to the background plasma can cause the development of fast electrostatic collective instabilities. In this paper we examine numerically the defocusing effects of two-stream instability on the ion beam propagating in neutralizing background plasma. The scaling laws for the average de-focusing forces on the beam ions are identified, and confirmed by comparison with numerical simulations. These scalings can be used in the development of realistic ion beam compression scenarios in present and next-generation ion-beam-driven experiments.
\end{abstract}

\section{INTRODUCTION}

To achieve energy densities necessary for ion-beam-driven high energy density physics and heavy ion fusion applications, the intense ion beam pulse must be compressed transversely and longitudinally before it reaches the target. To achieve maximum compression, the intense ion beam pulse must propagate through background plasma which neutralizes the beam space-charge and provide a significant neutralization of the beam current, leading to almost ballistic focusing of the intense ion beam with radius $r_{b}$ much larger than the collisionless plasma skin depth $\lambda_{p}=c / \omega_{p}$, where $\omega_{p}=$ $\left(4 \pi e^{2} n_{p} / m_{e}\right)^{1 / 2}$ is the background electron plasma frequency and $n_{p}$ is the plasma density. For a thin, infinitely massive ion beam with $r_{b} \lesssim \lambda_{p}$ the beam current density is only partially neutralized, so that the remaining current density produces an azimuthal self-magnetic field that pinches the beam [1]. On the other hand the streaming of the moderately heavy ions relative to the background plasma can cause the development of fast electrostatic collective instabilities between beam ions and background electrons [2]. As demonstrated numerically in this paper using the particle-in-cell code LSP [3], the nonlinear stage of these instabilities produces fluctuating electrostatic fields which cause a significant drag on the background plasma electrons and result in producing local current densities which may significantly exceed the beam current density [4]. These overneutralizing background electron current densities reverse the beam self-magnetic field and lead to beam transverse defocusing. At the same time, the ponderomotive force of the unstable wave pushes background electrons transversely away from the unstable region inside the beam, which creates an ambipolar electric field, which also leads to ion beam transverse defocusing.

\section{NUMERICAL SIMULATIONS OF ION BEAM PROPAGATION IN NEUTRALIZING PLASMA}

To benchmark the scaling laws for the nonlinear defocusing forces, we simulated the propagation of hydrogen and lithium ion beams of different duration and densities in a background plasma of

\footnotetext{
${ }^{\mathrm{a}}$ e-mail: estarts@pppl.gov, research supported by the U.S. Department of Energy.
}

This is an Open Access article distributed under the terms of the Creative Commons Attribution License 2.0, which permits unrestricted use, distribution, and reproduction in any medium, provided the original work is properly cited. 

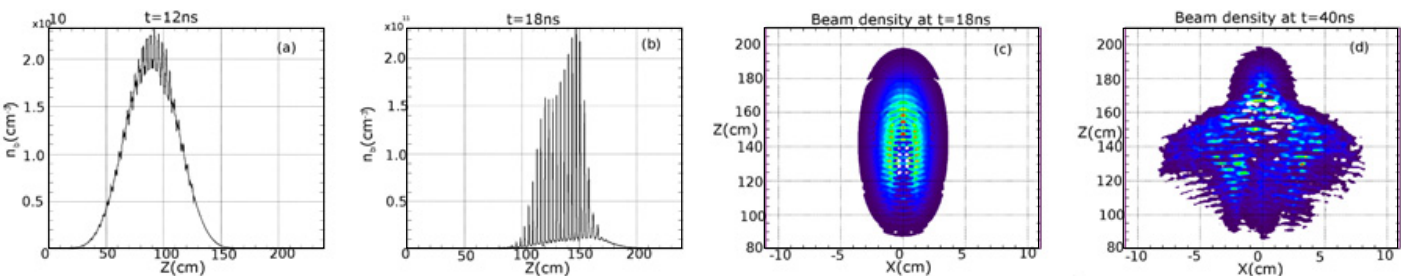

Figure 1. Longitudinal beam density profile at $t=12 \mathrm{~ns}$ (a) and $t=18 \mathrm{~ns}$ (b) and color plots of beam density at $t=18 \mathrm{~ns}(\mathrm{c})$ and $t=40 \mathrm{~ns}(\mathrm{~d})$.
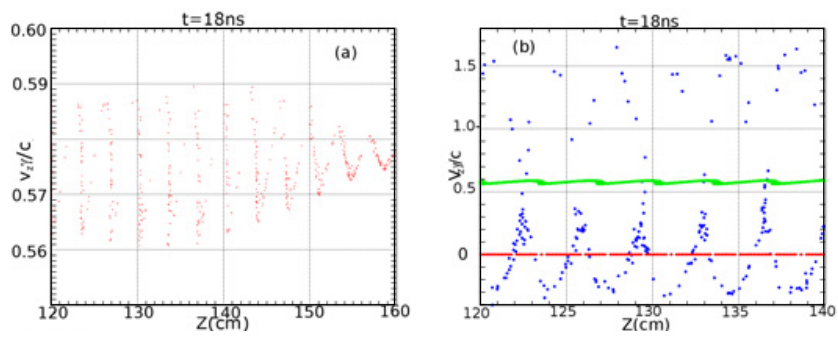

Figure 2. Beam (red) (a) and background electrons (blue) (b) phase-space $z-v_{z} \gamma$ at $t=18 \mathrm{~ns}$.

different densities. The hydrogen beam simulations were carried out for the following parameters: the ion beam has a Gaussian density profile and pulse duration $T=12 \mathrm{~ns}$, with beam velocity $v_{b}=c / 2$, where $c$ is the speed of light in vacuo; the beam density is $n_{b}=2 \times 10^{10} \mathrm{~cm}^{-3}$, and the beam radius is $r_{b}=2 \mathrm{~cm}$; the beam propagates through a stationary, singly-ionized carbon plasma with plasma density $n_{p}=2 \times 10^{11} \mathrm{~cm}^{-3}$. The results of the simulations are illustrated in Fig. 1 . At time $t=0$ the ion beam enters the plasma at $Z=0$. As shown in Fig.1(a), at time $t=12 \mathrm{~ns}$ the two-stream instability between the beam ions and the plasma electrons begins to develop and is completely developed to the saturation level by time $t=18 \mathrm{~ns}$ [Fig. 1(a)]. At this time the hydrogen ion beam density is modulated with density variations of order $100 \%$ of the original beam density. The longitudinal phase space of the beam ions at $t=18 \mathrm{~ns}$ is shown in Fig. 2(a) and plasma electrons in Fig. 2(b)(blue dots). Note that at the time of saturation at $t=18 \mathrm{~ns}$, the beam ions begin to be trapped by the nonlinear wave while the background electrons are completely trapped by the wave and perform nonlinear oscillations with amplitude of the velocity oscillations $v_{m}^{e} \sim v_{b}$. Figure 3 shows the azimuthal magnetic field and the longitudinal current density. As shown in Figs. 3(a) and 3(b), at $t=12 \mathrm{~ns}$ the beam current density is partially neutralized by the return current of the background plasma electrons. The resulting net current density produces magnetic field that results in a focusing force which leads to beam pinching. But as the instability develops and saturates at $t=18 \mathrm{~ns}$, the longitudinal current density and magnetic field change sign [Fig. 3(c) and 3(d)]. The resulting reversed magnetic field associated with a net current density leads to a defocusing of the ion beam pulse. The average radial component of the electric field (averaged over the fluctuations) at the time when the instability saturates at $t=18 \mathrm{~ns}$ is shown in Fig. 4. The large average electric field has an ambipolar structure and also contributes to the ion beam defocusing. Figures 1(c) and 1(d) show the ion beam density at two instants of time, $t=18 \mathrm{~ns}$ and $t=40 \mathrm{~ns}$. Note that after $40 \mathrm{~ns}$ the part of the beam where the instability has significantly developed is completely defocused, while the beam head remains relatively unchanged.

Similar simulations have also been carried out for parameters relevant to upcoming NDCX-II experiments [5]. In these simulations a singly-ionized lithium ion beam with velocity $v_{b}=c / 30$ propagates 

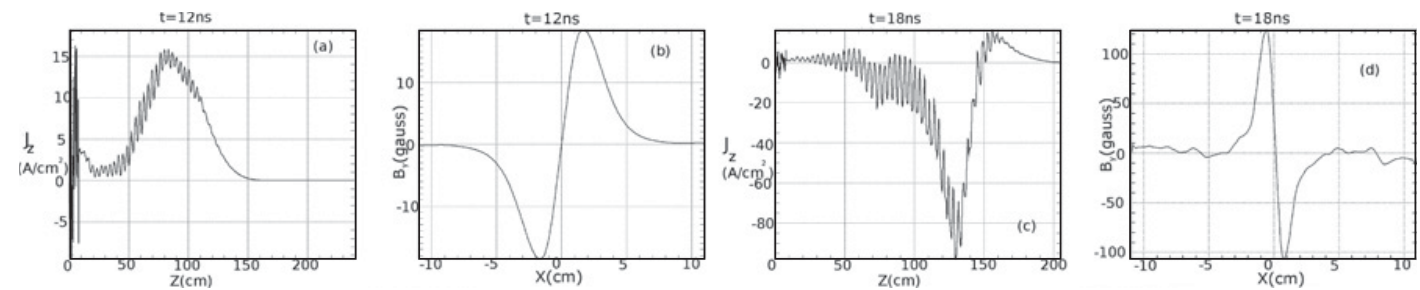

Figure 3. Current density profile at $r=0$ in (a) and (c), and the transverse profile of the associated azimuthal magnetic field in the middle of the beam in (b) and (d), at $t=12 \mathrm{~ns}$ in (a) and (b), and $t=18 \mathrm{~ns}$ in (c) and (d).
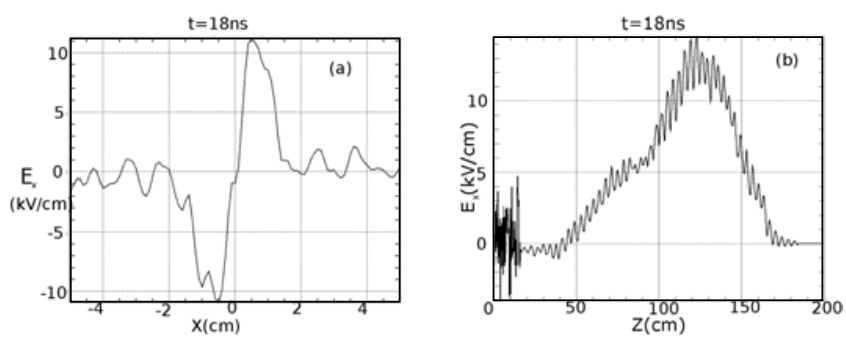

Figure 4. Average radial electric field profiles: (a) transverse profile at the middle of the beam, and (b) longitudinal profile at $r=0.75 \mathrm{~cm}$.

through a neutralizing singly-ionized background carbon plasma with density $n_{p}=0.55 \times 10^{11} \mathrm{~cm}^{-3}$. At the entrance into the plasma, the beam density is $n_{b}=2 \times 10^{9} \mathrm{~cm}^{-3}$, the beam radius is $r_{b}=1.41 \mathrm{~cm}$ and the beam pulse duration is $T=20 \mathrm{~ns}$. After two meters of propagation at $t=200 \mathrm{~ns}$ the same two-stream instability is observed to develop and saturate, which results in longitudinal beam density variations of order $90 \%$ of the original beam density. The unstable electric field fluctuations reverse the magnetic field, but the nonlinearly generated defocusing fields do not have enough time to significantly defocus the beam.

\section{DISCUSSION AND ANALYTICAL ESTIMATES}

In this section we make simple estimates of the nonlinearly generated transverse defocusing fields based on the physical picture observed in the simulations. The action of the electrostatic field of the unstable wave with phase velocity $\omega / k_{z} \approx v_{b}$ produces an average longitudinal electron current density

$$
\begin{aligned}
<J_{z}^{e}> & =J_{z}^{i n d}-e<\delta n^{e} \delta v_{z}^{e}>=-e n_{p}<v_{z}^{e}>-e \frac{n_{p}}{v_{b}}<\left(\delta v_{z}^{e}\right)^{2}> \\
& =-e n_{p}<v_{z}^{e}>-\frac{1}{2} \frac{n_{p}}{n_{b}}\left(\frac{v_{m}^{e}}{v_{b}}\right)^{2} J_{b},
\end{aligned}
$$

where $v_{m}^{e}$ is the velocity oscillation amplitude of the plasma electrons in the wave and $J_{z}^{\text {ind }}=-e n_{p}$ $\left\langle v_{z}^{e}\right\rangle$ is the average longitudinal electron current produced by the longitudinal inductive electric field which acts to reduce a current density $<J_{z}^{n o n}>+J_{z}^{b}$, consisting of non-linear current density driven by the electrostatic wave and the beam current density $J_{z}^{b}$, by a factor $1 /\left(1+r_{b}^{2} \omega_{p}^{2} / c^{2}\right)$. The total enhanced 
return current density

$$
<J_{z}>=J_{z}^{b}+<J_{z}^{e}>=\frac{J_{z}^{b}}{\left(1+r_{b}^{2} \omega_{p}^{2} / c^{2}\right)}\left[1-\frac{1}{2} \frac{n_{p}}{n_{b}}\left(\frac{v_{m}^{e}}{v_{b}}\right)^{2}\right],
$$

can reverse average magnetic field

$$
<B_{y}>=\frac{4 \pi}{c}<J_{z}>r_{b} \sim \frac{2 \pi e n_{b} r_{b} \beta_{b}}{\left(1+r_{b}^{2} \omega_{p}^{2} / c^{2}\right)}\left[1-\frac{1}{2} \frac{n_{p}}{n_{b}}\left(\frac{v_{m}^{e}}{v_{b}}\right)^{2}\right],
$$

if $v_{m}^{e} / v_{b}>\left(2 n_{b} / n_{p}\right)^{1 / 2}$. The ponderomotive pressure of the unstable wave pushes electrons away from the unstable region inside the beam which sets up an ambipolar transverse electric field

$$
e<E_{x}>\sim m_{e} \frac{\left(v_{m}^{e}\right)^{2}}{r_{b}}
$$

To estimate the value of $v_{m}^{e} / v_{b}$ we note, that the unstable wave grows until plasma electrons start to oscillate with velocity amplitude of order the beam velocity $v_{m}^{e} \sim \omega / k_{z} \approx v_{b}$, or the beam ions begin to oscillate with velocity amplitude $v_{m}^{b}=v_{b}-\omega / k_{z} \sim \gamma / k_{z} \approx\left(\gamma / \omega_{p}\right) v_{b}$. Here $\gamma$ is the linear instability growth rate $\gamma \sim \omega_{p}\left(\omega_{b} / \omega_{p}\right)^{2 / 3}$ and velocity amplitudes of ions and electrons are related as $v_{m}^{e}=$ $\left(m_{b} / m_{e}\right) v_{m}^{b}\left(\omega-k_{z} v_{b}\right) / \omega \sim\left(m_{b} / m_{e}\right) v_{m}^{b}\left(\gamma / \omega_{p}\right)$. Therefore, the amplitude of background electrons velocity oscilations at the saturation can be estimated as

$$
\left(\frac{v_{m}^{e}}{v_{b}}\right) \sim \min \left[\left(\frac{n_{b}}{n_{p}}\right)^{2 / 3}\left(\frac{m_{b}}{m_{e}}\right)^{1 / 3} ; 1\right] .
$$

The first limit corresponds to the instability saturated by the beam ion trapping, and the second is the saturation due to trapping of plasma electrons.

Knowing the defocusing forces on the ion beam, we can estimate the defocusing time $T$ defined as time it takes the beam radius to double after the instability has developed and saturated, i.e, $\Delta r_{b} / r_{b}=1$ and defocusing propagation distance $L=v_{b} T$

$$
T \sim\left(\frac{r_{b}}{v_{b}}\right) \frac{\left(m_{b} / m_{e}\right)^{1 / 2}}{v_{m}^{e} / v_{b}}, \quad L \sim r_{b} \frac{\left(m_{b} / m_{e}\right)^{1 / 2}}{v_{m}^{e} / v_{b}} .
$$

For the large beam densities, $n_{b} / n_{p}>\left(m_{e} / m_{b}\right)^{1 / 2}$, when $v_{m} / v_{b} \sim 1$ and instability is saturated by background electrons trapping, the defocusing time and distance are

$$
T \sim\left(\frac{r_{b}}{v_{b}}\right)\left(\frac{m_{b}}{m_{e}}\right)^{1 / 2}, \quad L \sim r_{b}\left(\frac{m_{b}}{m_{e}}\right)^{1 / 2} .
$$

For low density beams with $n_{b} / n_{p}<\left(m_{e} / m_{b}\right)^{1 / 2}$, the saturation is by the beam ions trapping, and the defocusing time and distance are

$$
T \sim\left(\frac{r_{b}}{v_{b}}\right)\left(\frac{n_{p}}{n_{b}}\right)^{2 / 3}\left(\frac{m_{b}}{m_{e}}\right)^{1 / 6}, L \sim r_{b}\left(\frac{n_{p}}{n_{b}}\right)^{2 / 3}\left(\frac{m_{b}}{m_{e}}\right)^{1 / 6} .
$$

For the simulations of ion beam propagation in the plasma with parameters given in Sec. 2, the defocusing time is $T \sim 6 \mathrm{~ns}$ and $T \sim 160 \mathrm{~ns}$ for a proton beam and a lithium beam respectively, which is in qualitative agreement with the simulations. Note that total defocusing time observed in simulations also includes the time for instability to grow and saturate. 


\section{References}

[1] I. D. Kaganovich, G. Shvets, E. A. Startsev and R. C. Davidson, Phys. Plasmas 8, 4180 (2001)

[2] R. C. Davidson, M. Dorf, I. Kaganovich, H. Qin, E. A. Startsev, S. M. Lund, Nuclear Instruments and Methods in Physics Research A606, 11 (2009)

[3] D. R. Welch, D. V. Rose, B. V. Oliver, R. E. Clark, Nuclear Instruments and Methods in Physics Research A464, 134 (2001)

[4] R. N. Sudan, Phys. Rev. Lett. 37, 1613 (1976)

[5] A. Friedman et. al., Phys. Plasmas 17, 056704 (2010) 